\title{
Radiological changes among artistic gymnasts in Gauteng Province
}

A Geldenhuys-Koolen, ${ }^{1}$ BSc (Physiotherapy), MSc (Med) (Exercise Science); D Constantinou, ${ }^{2}$ MB BCh, BSc (Med) (Hons), MSc (Med), FFIMS; Y Coopoo, ${ }^{2}$ DPhil, FACSM

\author{
${ }^{1}$ Private practice, Adele Geldenhuys Physiotherapy, Johannesburg, South Africa \\ ${ }^{2}$ Centre for Exercise Science and Sports Medicine, School of Therapeutic Sciences, Faculty of Health Sciences, University of the Witwatersrand, \\ Johannesburg, South Africa
}

Corresponding author: A Geldenhuys-Koolen (adele@koolcon.co.za)

Background. The skeletal immaturity of competitive female gymnasts allows for a unique physiological predisposition to injuries as a result of the spine, limbs, ankles and wrists still growing. Studies have shown that lower back (spinal) injuries account for approximately $12 \%$ of injuries in female gymnasts.

Objectives. The primary objective of the study was to determine the prevalence of radiological changes in female artistic gymnasts in South Africa. A further objective was to determine whether these radiological changes were associated with symptoms and with the amount of time spent training.

Methods. A sample of 40 female artistic gymnasts with a mean of age 15.2 years (range 10 - 31) was included in the study. Thirty-one were active gymnasts and nine were retired at the time of the current study. Measuring instruments included questionnaires and X-rays. Results. X-ray analysis of symptomatic versus asymptomatic gymnasts showed no significant differences. Of the 18 gymnasts training $<25 \mathrm{~h} /$ week, 13 (72\%) had degenerative changes detectable by X-ray. Of the 22 gymnasts training $>25 \mathrm{~h} /$ week, 15 (68\%) had degenerative changes detected by X-ray. Radiological changes were higher than those in other studies.

Conclusion. The prevalence of radiological changes was higher than international norms, however there was little difference between symptomatic and asymptomatic gymnasts. Patient self-reports of symptoms had little value in diagnosing change in the lumbar spine. Training duration affected the prevalence of changes in the lumbar spine and could be related to conditioning and experience.

S Afr J SM 2014;26(3):69-72. DOI:10.7196/SAJSM.532

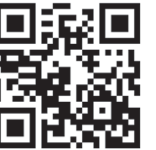

In South Africa (SA), women's gymnastics participation is on the increase. ${ }^{[1]}$ Competition season for Level 1 - 4 gymnasts starts in March and ends in June, whereas Level 5 and higher gymnasts start their competitions in June and continue through to September. The Junior and Senior Olympic entry female gymnasts start with testing competitions as early as January, and the first major competition takes place in February. Qualification trials for world championships and the All Africa Games take place in SA throughout the year. The gymnasts are also selected for numerous international competitions based on their performances and their rankings with the SA Gymnastics Federation.

Gymnastics appears to be associated with a high incidence of injury when compared with most other sporting activities, ${ }^{[2]}$ with the amount of impact applied to the body relatively high in comparison with other sports. ${ }^{[3]}$ The skeletal immaturity of gymnasts also allows for a unique physiological predisposition to injuries because the spine, limbs, ankles and wrists are still growing. Studies have shown that lower back (spinal) injuries account for approximately $12 \%$ of injuries in women's gymnastics. ${ }^{[4]}$ Published case studies show that back injuries tend to have a gradual onset, and involve predominantly advanced-level gymnasts. These studies have also indicated that an increase in skill and competitive level are risk factors for injury. ${ }^{[4]}$

Sports-related injuries are of two types. Macrotrauma occurs as an acute, perhaps dramatic, event such as a concussion, spinal cord injury, fracture or dislocation. Microtrauma occurs as a repeated injury, usually not noticed initially because the injury is microscopic in magnitude, but in which the cumulative trauma leads to pain and, in some cases, significant disability, as occurs with stress fractures.

Although any number of injuries may occur in all sports, some injuries are recognised to be particularly prevalent in specific sports. ${ }^{[5]}$ In gymnastics, spondylolysis occurs frequently due to the hyperextended positions and rotational forces in gymnastic routines, e.g. back walk-overs. ${ }^{[6]}$ Spondylolysis may represent a form of stress fracture. Prevention includes abdominal and spinal muscle strengthening. However, more recent evidence regarding specific prevention and treatment is required; the findings in this study may lead to preventive measures.

A 2005 study by Cohen and Stuecker ${ }^{[7]}$ showed the importance of detecting and monitoring the early onset of spondylolysis due to degeneration of the pars interarticularis of the vertebrae. This condition is not isolated to gymnastics; however, it has a higher prevalence in sports where the lumbar spine is placed under stress in hyperextension positions, i.e. gymnastics, ballet, swimming (butterfly) or in unilateral sports such as bowling in cricket. ${ }^{[6]}$

Imaging studies reported in the medical literature have shown that degenerative disc disease and spinal injuries are more frequent in competitive female gymnasts than in asymptomatic non-athletic people of the same age. ${ }^{[8]}$ Although these findings are more prevalent in the competitive gymnast, other studies suggest that they may not 
be of clinical significance. A study documenting magnetic resonance imaging (MRI) findings in symptomatic and asymptomatic Olympic gymnasts in the USA, reported that symptomatic patients exhibited radiological changes absent in asymptomatic patients. ${ }^{[9]}$

It is accepted that X-rays may not be appropriate for assessing detail of lumbar spine pathology, but are sufficient for initial evaluation or when recommended by 'red flags. ${ }^{\text {'[1] }}$ The focus of this study was on X-rays rather than MRI because MRI scans in SA are costly and impractical, especially for the screening of asymptomatic subjects. Generally in SA, MRI scans are usually performed if the patient is symptomatic and when X-rays show radiological changes and further investigation is indicated. As underlying pathology detected on MRI scans may not be the cause of lower back pain, the value of MRI scans in refining epidemiological case definitions for lower back pain is limited. ${ }^{[11]}$ The detection of spondylolysis, not uncommon in gymnasts, is most sensitively detected by X-rays; ${ }^{[12]}$ therefore, X-ray imaging plays an important role in this. ${ }^{[13]}$ The review by Davis et al. ${ }^{[10]}$ states: 'The availability of equipment or personnel may influence the selection of appropriate imaging procedures or treatment'. Lack of financial resources is an influencing factor in the selection of imaging procedure, and an SA study reviewing the complementary roles of radiology and nuclear medicine stated that cost is an important consideration. ${ }^{[14]}$ In Africa, financial constraints and physical access to MRI imaging may be improving but is still limited, while $\mathrm{X}$-rays are more readily available. ${ }^{[15,16]}$ As such, the use of X-rays in the context of assessing radiological findings and lower back pain in the African context is currently appropriate. ${ }^{[17]}$

Owing to the lack of recent research on X-ray changes in gymnasts and the questions relating to the clinical significance of these changes, there is a need to investigate the prevalence of radiological changes in female artistic gymnasts in SA, and to correlate these with symptoms. It is also important to note that, to date, there has been no research conducted on artistic gymnasts in SA because the sport has only recently become popular. ${ }^{[1]}$ To date, there have been no radiological studies conducted on artistic gymnasts in $\mathrm{SA},{ }^{[1]}$ nor is there current research on radiological changes among the broader gymnast population.

The study was approved by the faculty postgraduate studies committee, and ethics approval was granted by the university Human Research Ethics Committee (Medical). Questionnaires and radiological tests were used to investigate history, type of training, experience and injuries sustained in the participating gymnasts. One of the benefits of this study was ascertaining the state of spinal health of this group of gymnasts. Based on the results, group exercise programmes could be created to prevent further back injuries, and to prevent progression of back injuries where they exist.

\section{Methodology}

Radiological imaging of the participating gymnasts was performed with anteroposterior, lateral and oblique views. The X-rays were examined and reported on by a single radiologist. The radiologist transcribed the findings on a standardised reporting form. Once all X-rays were examined and reported on, they were reviewed by a second independent radiologist, who, using the same criteria, reexamined and reported on the films. Both radiologists reached the same conclusions.

\section{Sample design}

Owing to the small population size, a census was more practical than drawing a sample. An attempt was made to conduct a census of all currently active elite gymnasts registered with clubs in Gauteng, specifically those falling into the subcategory of artistic gymnasts. Of the 20 registered gymnastics clubs in Gauteng, seven clubs offered a high-performance programme. Four of these clubs did not have any elite gymnasts at the time of the study. The remaining three clubs were included in the study. Once consent was obtained, participants were given a questionnaire to complete. The participants were able to take the questionnaires home to complete with the help of their parent/ guardian.

All female artistic gymnasts born between 1990 and 2000, and who were currently training $\geq 15 \mathrm{~h} /$ week for a minimum of 3 years, were considered for inclusion. The training programme had to be a highperformance programme. Only gymnasts who consented, or in the case of minors who assented and whose parents consented for their participation, were included in the study. In total, 31 currently active artistic gymnasts were included in the study, while four qualifying gymnasts refused to participate, yielding a response rate of $89 \%$.

A cohort of nine former elite-level gymnasts who had retired from the sport was also included in the study in order to gather radiological data for comparison with the currently active group. There was no age limit for the retired group; the only requirement being that they had trained for at least 3 years, and for $\geq 15 \mathrm{~h} /$ week in a high-performance programme.

\section{Measuring instruments \\ Questionnaires}

Questionnaire content validity was ensured by obtaining input from gymnasts, while test-retest reliability was also assessed, achieving $100 \%$ agreement between both tests.

\section{$X$-rays}

$\mathrm{X}$-rays were taken by three radiographers, following the same procedures for each view. The X-ray machines used were the Phillips Omnidiagnost, Siemens Iconos 100 and Siemens Sieragraph B. The films were processed in a Konical Minolta SRX201, using Fujifilm HR U30. The X-rays were taken in the following views, using the procedure as set out in Clark's Positioning in Radiography. ${ }^{[18]}$ Each subject underwent five lumbar spine X-ray views, namely anteroposterior (AP), lateral, oblique, stress views (extension and flexion) and lateral coned L5, S1 views.

\section{Results}

Descriptive statistics were used to analyse the data collected using questionnaires, as well as the data resulting from the radiological analysis.

In total, $90 \%$ of gymnasts reported a peripheral injury, while $50 \%$ reported a back injury.

\section{Peripheral injuries: Self-reports}

Based on the data collected from the questionnaires, $90 \%$ of the 40 gymnasts reported having sustained a peripheral injury (all injuries excluding back injury). Prevalence rates among currently active gymnasts were similar to the retired group: 28 (90\%) of the 31 current 
gymnasts reported peripheral injuries, while $8(88 \%)$ of the 9 retired gymnasts reported peripheral injuries. Of the 36 gymnasts with peripheral injuries, 33 (92\%) of the injuries happened during training and $3(8 \%)$ during competition.

\section{Back injuries: Self-reports}

Of the 40 gymnasts who completed the questionnaire, 50\% reported sustaining a back injury, either before the time of data collection or before the time of retirement. Of the 20 gymnasts with back injuries, $75 \%$ reported the injuries to be located in the lumbar spine, with $15 \%$ located in the thoracic spine and $10 \%$ located in the cervical spine.

\section{Back injuries: X-rays}

Spinal X-rays were taken of each gymnast $(N=40)$ and analysed for the following changes: scoliosis, spondylolysis, spondylolisthesis and degenerative changes (disc and facet joint degeneration, osteophyte formation).

Among those who reported back injury, the most prevalent condition identified by radiological analysis was scoliosis (75\%), followed by degenerative changes (70\%) (Table 1). The least common change was spondylolysis. Interestingly, among those who did not report back injury, a high percentage was subsequently shown to have changes they were unaware of. A similar pattern emerged, with scoliosis and degenerative changes being most prevalent (Table 2).

In order to obtain an indication of whether the prevalence levels of this Gauteng study deviated substantially from international data, we compared our data with prevalence levels reported in a meta-analysis conducted by Caine and Nassar. ${ }^{[4]}$ Given the substantial variation from study to study, the lowest and highest values are reported to provide an indication of the range (Table 3). Prevalence rates reported internationally showed lower prevalence of scoliosis (both lowest and highest prevalences were below the prevalence rates in Gauteng). In contrast, in Gauteng, spondylolysis and spondylolisthesis had prevalence rates between the lowest and highest rates reported internationally.

\section{Training exposure time} and radiological changes

Gymnasts were allocated to either a low or high training exposure group. Since elite

Table 1. Prevalence of radiological changes, subject reported back injury $(N=20)$

\begin{tabular}{lllll}
\hline & Total, $\%$ & Total, $\boldsymbol{n}$ & Current, $\boldsymbol{n}$ & Retired, $\boldsymbol{n}$ \\
\hline Scoliosis & 75 & 15 & 11 & 4 \\
Degenerative changes & 70 & 14 & 11 & 3 \\
Spondylolysis & 15 & 3 & 2 & 1 \\
Spondylolisthesis & 20 & 4 & 3 & 1
\end{tabular}

Table 2. Prevalence of radiological changes, subject did not report back injury $(N=20)$

\begin{tabular}{lllll}
\hline & Total, \% & Total, $\boldsymbol{n}$ & Current, $\boldsymbol{n}$ & Retired, $\boldsymbol{n}$ \\
\hline Scoliosis & 75 & 15 & 12 & 3 \\
Degenerative changes & 85 & 17 & 12 & 5 \\
Spondylolysis & 15 & 3 & 1 & 2 \\
Spondylolisthesis & 15 & 3 & 2 & 1
\end{tabular}

Table 3. Prevalence of radiological changes, international studies

\begin{tabular}{lll}
\hline & Lowest reported, \% & Highest reported, \% \\
\hline Scoliosis & 11.5 & 19.2 \\
Spondylolysis & 9.1 & 32.8 \\
Spondylolisthesis & 3.3 & 15.4
\end{tabular}

gymnasts train for a minimum of $25 \mathrm{~h} /$ week, this was used as a cut-off point in order to evaluate the effect of training exposure on radiological changes. Gymnasts who trained $<25 \mathrm{~h}$ /week were allocated to the lowexposure group, while gymnasts who trained for $>25 \mathrm{~h} /$ week were allocated to the highexposure group. The results showed that gymnasts in the high-exposure group were less likely to have evidence of degenerative changes in the spine than those in the lowexposure group, with prevalence rates of $68 \%$ v. $72 \%$, respectively.

\section{Discussion}

Scoliosis, spondylolysis, spondylolisthesis and other signs of degenerative change were examined using X-rays. The results showed that there can be significant structural deficit in the lumbar spines of gymnasts, even without reports of pain or back injury. Differences in actual injury prevalence between symptomatic and asymptomatic gymnasts, as confirmed by X-ray, were small. This means that the presence or absence of symptoms is an unreliable indicator of injury. Further, this could mean that asymptomatic gymnasts may unknowingly expose themselves to excessive training and competition, leading to faster progression of changes. Therefore, imaging should be performed on gymnasts to monitor spinal changes and possibly initiate measures to prevent severe injury.

The findings of this study also showed that exposure time to gymnastics has an effect on the prevalence of back injuries. Back injuries occurred more in gymnasts who trained for $<25 \mathrm{~h} /$ week. However, the results of other studies have concluded that greater exposure to training was directly related to an increased risk of injury. ${ }^{[1]}$ This may be due to a non-linear relationship between exposure time and back injury prevalence, which the discretised measure of exposure used in this study was unable to detect. Further research should be conducted with larger samples for a more refined measure of exposure, with a larger number of exposure categories.

Results showed that gymnasts training and competing at higher levels experienced more back injuries. This was also found in retired gymnasts who competed at senior Olympic level. This correlates with findings of other studies $^{[1]}$ where older gymnasts had higher exposure frequencies. Whether age is another risk factor for injury, because gymnasts competing at higher levels are generally older, is unknown. 


\section{Study limitations}

Limitations include: (i) the small, localised population, limited to the Gauteng Province of SA; (ii) no consideration given to the role of genetic differences related to ethnic group; and (iii) the exact gymnastic routines were not described or distinguished, which can be seen as a limitation as different moves and routines could have different effects.

\section{Conclusion}

While it is well-known that degenerative conditions and other structural changes such as scoliosis, spondylolysis and spondylolisthesis are more prevalent within the gymnastic population than the general population, this has not been demonstrated among elite artistic gymnasts.

Symptoms based on self-reports are not reliable indicators of the presence of back injury. The use of X-rays in the context of limited resources and accessibility is appropriate in gymnasts with high volumes of training as both symptomatic and asymptomatic groups showed similar prevalence rates when using X-rays as the measuring instrument.

Therefore, it is important to evaluate radiological spinal changes in gymnasts who are training at high performance level and competing professionally, both in symptomatic and asymptomatic gymnasts. The timing of when this should be done is not entirely clear. The importance of proper rehabilitation after injury and before return to high-level activity is also highlighted to prevent further injury and reduce the rate of early retirement from the sport. Proper conditioning of gymnasts is needed to prevent injury during skill acquisition and performance.

Training duration affects the prevalence of changes in the lumbar spine; however, the results contradict prior research, suggesting further research is required.

\section{References}

1. Adamson I. Gymnastics injuries: A quantitative profile of athletes in the greater Durban area. Dissertation submitted in partial compliance with the requirements for a Master's Degree in Technology: Chiropractic in the Department of Chiropractic at the Durban Institute of Technology. 2006. http://www.dut.ac.za (accessed 3 March 2014).
2. Kolt G, Kirkby R. Injury in Australian female competitive gymnasts: A psychological perspective. Aust J Physiother 1996;42(2):121-126. [http://dx.doi.org/10.1016/S0004 9514(14)60444-X]

3. Daly RM, Bass SL, Finch CF. Balancing the risk of injury to gymnasts: How effective are the counter measures? Br J Sports Med 2001;35(1):8-18. [http://dx.doi. org/10.1136/bjsm.35.1.8]

4. Caine DJ, Nassar L. Gymnastic injuries. Med Sport Sci 2005;48:18-58. [http://dx.doi org/10.1159/000084282

5. Boden BP, Osbahr DC, Jimenez C. Low-risk stress fractures. Am J Sports Med 2001;29(1):100-111.

6. Bruggeman GP. Mechanical load in artistic gymnastics and its relation to apparatus and performance. In: Leglise M, ed. Symposium Medico-Technique. Lausanne, Switzerland: International Gymnastics Federation, 1999:17-27.

7. Cohen E, Stuecker RD. Magnetic resonance imaging in diagnosis and followup of impending spondylolysis in children and adolescents: Early treatment may prevent pars defects. J Pediatr Orthop B 2005;14(2):63-67. [http://dx.doi. org/10.1097/01202412-200503000-00001]

8. Swärd L, Hellstrom M, Jacobsson B, Pëterson L. Back pain and radiologic changes in the thoraco-lumbar spine of athletes. Spine 1990;15(2):124-129. [http://dx.doi org/10.1097/00007632-199002000-00015]

9. Bennett DL, Nassar L, DeLano, MC. Lumbar spine MRI in the elite-level female gymnas with low back pain. Skeletal Radiol 2006;35(7):503-509. [http://dx.doi.org/10.1007/ s00256-006-0083-7]

10. Davis PC, Wippold FJ, Brinberg JA, et al. ACR Appropriateness Criteria on low back pain. J Am Coll Radiol 2009;6(6):401-407. [http://dx.doi.org/10.1016/j. jacr.2009.02.008]

11. Endean A, Palmer KT, Coggon D. Potential of magnetic resonance imaging findings to refine case definition for mechanical low back pain in epidemiological studies: A systematic review. Spine 2011;36(2):160-169. [http://dx.doi.org/10.1097/ BRS.0b013e3181cd9adb]

12. Tallarico RA, Madom IA, Palumbo MA. Spondylolysis and spondylolisthesis in the athlete. Sports Med Arthrosc Rev 2008;16(1):32-38. [http://dx.doi.org/10.1097/ JSA.0b013e318163be50]

13. Keller MS. Gymnastics injuries and imaging in children. Pediatr Radiol 2009;39(12):12991306. [http://dx.doi.org/10.1007/s00247-009-1431-2]

14. Warwick J, Lotz J. Integrated imaging: The complementary role of radiology and nuclear medicine. South African Journal of Radiology 2013;17(4):149-153. [http://dx.doi org/10.7196/sajr.1033]

15. Ogbole GI, Adeleye AO, Adeyinka AO, Ogunseyinde OA. Magnetic resonance imaging Clinical experience with an open low-field-strength scanner in a resource challenged African state. J Neurosci Rural Pract 2012;3(2):137-143. [http://dx.doi.org/10.4103/09763147.98210]

16. Potchen MJ, Kampondeni S, Birbeck GL, et al. Magnetic resonance imaging in Malawi: Contributions to clinical care, medical education and biomedical research. Malawi Med J 2011;23(2):62-66. [http://dx.doi.org/10.4314/mmj.v23i2.70753

17. Igbinedion BOE, Akhigbe A. Correlations of radiographic findings in patients with low back pain. Nig Med J 2011;52(1):28-34.

18. Whitley AS, Sloane C, Hoadley G, Moore AD. Clarke’s Positioning in Radiography. 12th ed. London: Hodder Arnold, 2005. 